\title{
Future of Radiotherapy Delivery in Rectal Cancer- European and US Approaches
}

\author{
Jordan A Torok, MD, ${ }^{1}$ Brian G Czito, MD, ${ }^{2}$ Christopher G Willett, $\mathrm{MD}^{3}$ and Manisha Palta, MD ${ }^{4}$ \\ 1. Resident; 2. Associate Professor; 3. Professor and Chair; 4. Assistant Professor, \\ Department of Radiation Oncology, Duke University Medical Center, Durham, North Carolina, US
}

\begin{abstract}
Neoadjuvant radiation therapy is integral in the management of patients with localized rectal cancer. In parts of Europe, patients with operable rectal cancer are treated with short-course radiation therapy delivered in five daily, 5 Gy fractions to a total dose of 25 Gy, followed by surgery within 1 week. In the US, the standard for locally advanced rectal cancer is neoadjuvant chemoradiotherapy. This approach is principally based on the results of the German Rectal Cancer Study Group trial evaluating preoperative compared with postoperative chemoradiation. Surgery is typically performed at 4-8 weeks following completion of long-course chemoradiotherapy, facilitating tumor downstaging, and potential sphincter sparing surgery. No significant difference in clinical outcomes has been observed between these two approaches in two randomized clinical trials; however, further follow-up of these studies and new results from ongoing trials are anticipated to further clarify the optimal neoadjuvant treatment strategy.
\end{abstract}

\section{Keywords}

Rectal cancer, radiation therapy, short course, long course, chemoradiation, neoadjuvant therapy

Disclosure: Jordan A Torok, MD, Brian G Czito, MD, Christopher G Willett, MD, and Manisha Palta, MD, have no conflicts of interest to disclose. No funding was received in the publication of this article.

Received: July 31, 2014 Accepted: November 2, 2014 Citation: Oncology \& Hematology Review, 2014;10(2):139-43 DOI: 10.17925/OHR.2014.10.2.139

Correspondence: Manisha Palta, MD, Department of Radiation Oncology, Duke University, DUMC 3085 Durham, NC 27710, US. E: manisha.palta@dm.duke.edu

Over the past several decades, two paradigms have emerged in the preoperative treatment of rectal cancer: a short course of radiation therapy (RT) often utilized in centers across Northern Europe and longcourse chemoradiation (CRT) in other parts of Europe and the US. The short-course technique was validated by randomized trials comparing this technique to surgery alone. The majority of these trials included patients with resectable, nonmetastatic rectal cancer, both early and advanced stages, with surgery 1 week following treatment completion. By contrast, long-course therapy is typically offered to patients with more advanced disease (cT3-4, N+) to improve local control, RO resection rates, and potentially increase rates of sphincter-preserving surgery. Randomized studies have compared long-course CRT followed by surgery to upfront surgery and postoperative CRT. Multiple studies have attempted to optimize the long-course CRT platform with the addition of oxaliplatin. Two randomized trials have compared short-course RT to long-course CRT; however, significant debate still exists regarding the optimal neoadjuvant approach in patients with rectal cancer.

\section{Short-course Radiotherapy}

In early trials of preoperative short-course RT from Sweden, patients were randomized to preoperative RT (25 Gy in five daily fractions over 5-7 days) or underwent surgery alone. The incidence of pelvic recurrence was significantly reduced with RT at the expense of increased postoperative morbidity. ${ }^{1,2}$ A similar follow-up trial with improved RT techniques showed reduction in both locoregional and distant recurrence as well as improvement in overall survival (OS) with the short-course regimen. ${ }^{3}$ The rate of postoperative morbidity was reduced with the improved RT technique and postoperative mortality was low in both groups. These trials set the stage for the Swedish Rectal Cancer Trial, which randomized patients to the short-course regimen followed by surgery 1 week later versus surgery alone. After a median follow-up of 13 years, RT significantly reduced local recurrence $(\mathrm{LR})$ rates (9 versus $26 \% ; \mathrm{p}<0.001$ ) and improved OS (38 versus $30 \% ; p=0.008) .{ }^{4}$ Subset analyses found statistically significant reductions in LR for all stage groups. However, these LR and OS benefits were not without potential toxicity. With long-term follow-up, patients treated with RT were more likely to develop small bowel obstruction.5,6

The Swedish Rectal Cancer Trial was undertaken prior to widespread adoption of the total mesorectal excision (TME) surgical technique. Compared with the conventional surgical techniques at that time, TME significantly reduced LR rates. ${ }^{7}$ To determine the value of preoperative RT in the TME era, investigators from The Netherlands initiated a multicenter trial randomizing 1,861 patients to preoperative short-course RT with TME compared with TME alone. The RT dose/fractionation and surgical 
Table 1: Select Randomized Trials of Preoperative Short-course Radiotherapy

$\begin{array}{llll}\text { Trial } & \text { LR } & \text { DFS } & \text { OS } \\ \text { Swedish }(n=1,186)^{4} & (13 \text { year) } & (13 \text { year }) & (13 \text { year) } \\ \text { Surgery alone } & 26 \% & 62 \% & 30 \% \\ \text { RT + surgery } & 9 \%^{*} & 72 \%^{*} & 38 \% * \\ \text { Dutch }(n=1,861)^{8} & (10 \text { year) } & (10 \text { year) } & (10 \text { year) } \\ \text { TME alone } & 11 \% & 73 \% & 49 \% \\ \text { RT + TME } & 5 \%{ }^{*} & 80 \% & 48 \% \\ \text { MRC }(n=1,350)^{10} & (3 \text { year) } & (3 \text { year }) & (3 \text { year }) \\ \text { Surgery + select postop CRT } & 10.6 \% & 71.5 \% & 78.6 \% \\ \text { RT + TME } & 4.4 \% * & 77.5 \% * & 80.3 \%\end{array}$

*Statistically significant difference. CRT = chemoradiotherapy; DFS = disease-free survival; $L R=$ local recurrence; $M R C=$ Medical Research Council; OS = overall survival; $R T=$ radiotherapy; $T M E=$ total mesorectal excision .

Table 2: Randomized Trials of Postoperative versus Preoperative Chemoradiotherapy

\begin{tabular}{|c|c|c|c|c|c|}
\hline Trial & $\begin{array}{l}5 \text { year } \\
\text { LR }\end{array}$ & $\begin{array}{l}5 \text { year } \\
\text { DFS }\end{array}$ & $\begin{array}{l}5 \text { year } \\
\text { OS }\end{array}$ & pCR & $\begin{array}{l}\text { Sphincter } \\
\text { Preservation }\end{array}$ \\
\hline German $(n=823)^{11}$ & & & & & $\begin{array}{l}\text { (Judged to require } \\
\text { APR pre-treatment) }\end{array}$ \\
\hline Postop CRT & $13 \%$ & $65 \%$ & $74 \%$ & & $19 \%$ \\
\hline Preop CRT & $6 \% *$ & $68 \%$ & $76 \%$ & $8 \%$ & 39 \%* \\
\hline NSABP R-03 $(n=267)^{13}$ & & & & & (5 year) \\
\hline Postop CRT & $10.7 \%$ & $53.4 \%$ & $65.6 \%$ & & $24.2 \%$ \\
\hline Preop CRT & $10.7 \%$ & $64.7 \% *$ & $74.5 \%$ & $15 \%$ & $33.9 \%$ \\
\hline Korea $(n=240)^{14}$ & & & & & ( $<5 \mathrm{~cm}$ from verge) \\
\hline Postop CRT & $6 \%$ & $74 \%$ & $85 \%$ & & $42 \%$ \\
\hline Preop CRT & $5 \%$ & $73 \%$ & $83 \%$ & $17 \%$ & $68 \% *$ \\
\hline
\end{tabular}

*Statistically significant difference. $A P R=$ abdominoperineal resection; $C R T=$ chemoradiation; $D F S=$ disease-free survival; $L R=$ local recurrence; $N S A B P=$ National Surgical Adjuvant Breast and Bowel Project; $O S=$ overall survival; $P C R=$ pathological complete response.

timelines were similar to the Swedish trials. The most recent update with median follow-up of 12 years demonstrated a persistent reduction in the 10-year cumulative incidence of LR (5 versus $11 \%$; $p<0.0001) .{ }^{8}$ Compared to the Swedish Rectal Cancer Trial, overall LR rates were substantially lower with TME. The 10-year risk of LR with TME alone for stage I and III rectal cancers was $3 \%$ and $19 \%$, respectively. While there was no improvement in 10-year OS with RT (48 versus $49 \% ; p=0.86$ ), OS was improved in stage III patients with negative circumferential radial margins ( 50 versus $40 \% ; p=0.03$ ). The benefit in local control was at the expense of impaired bowel function and incontinence. ${ }^{9}$

Considering the potential for overtreatment in some rectal cancer patients, the Medical Research Council (MRC) and National Cancer Institute of Canada ( $\mathrm{NCIC}$ ) initiated a multicenter randomized trial comparing short-course preoperative RT (25 Gy/5) versus a selective postoperative CRT (45 Gy in 25 fractions with concurrent 5-fluorouracil [5FU]) approach for patients with an involved circumferential resection margin ( $\leq 1 \mathrm{~mm}$ ). TME was encouraged and performed in the majority of patients but not mandated by protocol. Unlike the former trials, modern staging techniques, including magnetic resonance imaging (MRI) and endoscopic ultrasound (EUS), were available (but not mandated) to determine operability and potentially guide resection. At a median follow-up of 4 years, the short-course RT arm was associated with a significantly lower 5-year LR (4 versus $11 \%$; hazard ratio [HR] 0.39 $95 \%$ confidence interval [Cl] 0.27-0.58; $\mathrm{p}<0.0001) .{ }^{10}$ Five-year disease-free survival (DFS) was also improved with short-course RT (74 versus $67 \%$; HR $0.7695 \% \mathrm{Cl} 0.62-0.94 ; \mathrm{p}=0.013)$, but there was no difference in 5-year OS (70 versus $68 \%$ ). Circumferential margin involvement was rare (12\%), and only $9 \%$ of patients in the selective postoperative group received adjuvant radiotherapy. The higher rates of LR in this group suggest postoperative CRT cannot fully compensate for an involved surgical margin, and/or the selection criteria for postoperative CRT were inadequate.

All three trials of preoperative short-course RT demonstrate significant reductions in LR (see Table 1). The latter two trials show exceedingly low rates of LR in stage I patients treated with TME with or without preoperative short-course RT. Considering the potential late effects of RT, these patients may not derive a clinically meaningful benefit from this approach. A potential disadvantage of early surgery following short-course RT is that 1 week is inadequate for tumor downstaging to occur. Such downstaging may allow patients with more distal tumors to undergo sphincter-sparing low anterior resection (LAR) instead of abdominoperineal resection (APR) and improve $\mathrm{R} 0$ resection rates. Another concern with the large doses per fraction used in the short-course RT regimen is the potential late gastrointestinal (GI) toxicity as seen with long-term follow-up from the Swedish and Dutch trials.

\section{Long-course Chemoradiotherapy}

In contrast to the experience with preoperative therapy in northern Europe, the historical standard for locally advanced rectal cancer in the US and other parts of Europe had been postoperative radiotherapy with or without chemotherapy. During the early to mid-1990s, multiple randomized trials were initiated centering on two major themes: long-course preoperative CRT versus postoperative CRT and long-course preoperative RT with or without chemotherapy. In a landmark trial, German investigators randomized 823 patients with cT3-4 or node-positive rectal cancer to receive 50.4 Gy in 28 fractions concurrent with continuous infusion 5FU, followed by TME surgery 6 weeks later, versus upfront TME and an identical CRT regimen, with an additional 5.4 Gy tumor bed boost. All patients were staged with modern imaging techniques) including EUS. Both arms were assigned to four cycles of adjuvant bolus 5FU following CRT completion. Initial results found improved compliance with preoperative therapy. A significant downstaging effect was seen with a pathologic complete response (PCR) rate of $8 \%$, lower rates of nodal positivity (25 versus $40 \%$ ), and higher rates of sphincter-preservation in patients judged to initially require an APR. ${ }^{11}$ Acute and late toxicity were reduced with the preoperative regimen. After a median follow-up of 11 years, 10-year LR was significantly lower with preoperative RT (7 versus $10 \%$; $p=0.048$ ). ${ }^{12}$ No difference was seen in DFS or OS.

The National Surgical Adjuvant Breast and Bowel Project (NSABP) R-03 had a similar design, although TME was not mandated. This trial closed prematurely due to poor accrual. Treatment results of 267 patients showed a similar effect of downstaging using preoperative therapy with a pCR rate of $15 \%$ and lower rates of nodal positivity. ${ }^{13}$ Five-year DFS was also significantly improved with preoperative RT (65 versus $53 \%$ ), but no significant difference in OS or LR was seen. A single-institution Korean trial with a similar design, but utilization of daily concurrent capecitabine, reported a pCR rate of $17 \%$ and improved rates of sphincter preservation (68 versus $42 \% ; p=0.008$ ) using preoperative therapy with no significant difference in 5-year LR, DFS, or OS. ${ }^{14}$ Taken together, these trials support 
Table 3: Select Randomized Trials of Various Long-course Chemoradiotherapy Regimens

\begin{tabular}{|c|c|c|c|c|c|}
\hline Trial & LR & DFS & OS & $\mathrm{pCR}$ & Sphincter Preservation \\
\hline Hofheinz et al. $(n=161 \#)^{20}$ & & & & & Sphincter-sparing surgery \\
\hline $5 F U$ & & & & $5 \%$ & $78 \%$ \\
\hline CAP & & & & $14 \%$ & $73 \%$ \\
\hline STAR-01 $(n=747)^{23}$ & & & & & APR rate \\
\hline $5 F U$ & & & & $16 \%$ & $21 \%$ \\
\hline $5 F U+O X$ & & & & $16 \%$ & $19 \%$ \\
\hline ACCORD 12/0405-Prodige $2(n=598)^{24,25}$ & 3 year & 3 year & 3 year & & APR rate \\
\hline CAP, 45 Gy RT & $6.1 \%$ & $67.9 \%$ & $87.6 \%$ & $13.9 \%$ & $24.8 \%$ \\
\hline $\mathrm{CAP}+\mathrm{OX}, 50$ Gy RT & $4.4 \%$ & $72.7 \%$ & $88.3 \%$ & $19.2 \%$ & $22.8 \%$ \\
\hline CAO/ARO/AIO-04 $(n=1,265)^{22,26}$ & & 3 year & & & APR rate \\
\hline $5 F U$ & & $71.2 \%$ & & $13 \%$ & $24 \%$ \\
\hline $5 F U+O X$ & & $75.9 \% *$ & & $17 \% *$ & $25 \%$ \\
\hline NSABP R-04 $(n=1,609)^{21}$ & & & & & Sphincter-sparing surgery \\
\hline $5 F U$ & & & & $17.8 \%$ & $59.4 \%$ \\
\hline CAP & & & & $20.7 \%$ & $59.3 \%$ \\
\hline $5 F U / C A P+O X$ & & & & $19.5 \%$ & $57.8 \%$ \\
\hline 5FU/CAP only & & & & $17.8 \%$ & $61 \%$ \\
\hline
\end{tabular}

\#Subgroup receiving neoadjuvant therapy; *statistically significant difference. $5 F U=5$-fluorouracil; $A P R=$ abdominoperineal resection; $C A P=C a p e c i t a b i n e ; ~ D F S=$ disease-free survival; $p C R=$ pathologic complete response; $L R=$ local recurrence; $N S A B P=$ National Surgical Adjuvant Breast and Bowel Project; OS = Overall survival; OX $=0 x a l i p l a t i n ; R T=$ radiotherapy.

Table 4: Randomized Trials of Short- versus Long-course Radiotherapy

\begin{tabular}{|c|c|c|c|c|c|c|c|}
\hline Trial & $\begin{array}{l}\text { Grade III/IV } \\
\text { Acute Toxicity }\end{array}$ & $\mathrm{pCR}$ & $\begin{array}{l}\text { Sphincter } \\
\text { Preservation }\end{array}$ & LR & DFS & OS & $\begin{array}{l}\text { Grade III/IV } \\
\text { Late Toxicity }\end{array}$ \\
\hline Polish trial $(n=312)^{27}$ & & & & (4 year) & (4 year) & (4 year) & (4 year) \\
\hline Short-course RT & $3.2 \%$ & $0.7 \%$ & $61.2 \%$ & $10.6 \%$ & $58.4 \%$ & $67.2 \%$ & $10.1 \%$ \\
\hline Long-course CRT & $18.2 \%$ & $16.1 \%$ & $58.0 \%$ & $15.6 \%$ & $55.6 \%$ & $66.2 \%$ & $7.1 \%$ \\
\hline Australian trial $(n=326)^{28}$ & & & & (3 year) & & (5 year) & (5 year) \\
\hline Short-course RT & $1.9 \%$ & $1 \%$ & $63 \%$ & $7.5 \%$ & NR & $74 \%$ & $5.8 \%$ \\
\hline Long-course CRT & $28 \%$ & $15 \%$ & $69 \%$ & $5.7 \%$ & NR & $70 \%$ & $8.2 \%$ \\
\hline
\end{tabular}

$\overline{C R T}=$ chemoradiation; $D F S=$ disease-free survival; $L R=$ local recurrence; $N R=$ not reported; $O S=$ overall survival; $P C R=$ pathologic complete response; $R T=$ radiotherapy.

the effect of CRT on enhancing tumor downstaging and improved rates of sphincter preservation (see Table 2).

During the same time period, investigators from the European Organisation for Research and Treatment of Cancer (EORTC) and the Fêdêration Francophone de Cancêrologie Digestive (FFCD) were conducting trials comparing longcourse preoperative RT with or without concurrent chemotherapy. The EORTC 22921 trial consisted of a $2 \times 2$ randomization of patients with cT3-4 resectable rectal cancer to 45 Gy preoperative RT with or without bolus 5FU and a second randomization to adjuvant 5FU versus observation. The addition of 5FU to RT resulted in a significantly higher pCR rate (5 vs $14 \%$ ) and reduced both tumor and nodal substages. ${ }^{15}$ Ten-year results were recently reported showing significant reductions in LR with the addition of chemotherapy. ${ }^{16}$ No significant differences were observed in distant metastases (DM), DFS or OS rates between the four arms. The FFCD 9203 trial randomized patients to preoperative RT or CRT and was identical to the EORTC trial, except all patients received adjuvant chemotherapy. Similar to the EORTC results, improved PCR (3 versus $15 \%$ ), lower rates of LR (17 versus $8 \%$ ), but no improvements in OS were seen with CRT. ${ }^{17}$ The addition of chemotherapy to radiation in unresectable T4 or previously unirradiated, locally recurrent rectal cancer has also been reported. In this trial, patients were treated to 50 Gy preoperative RT with or without bolus 5FU. CRT was associated with a higher RO resection
(84 versus $68 \%$ ) and pCR rate (16 versus $7 \%$ ). ${ }^{18}$ CRT reduced LR, DM, and improved 5-year cause-specific survival (5-year 72 versus $55 \%$ ). These trials support fluoropyrimidine-based CRT as the preferred long-course platform.

\section{Further Optimizing Long-course Chemoradiotherapy}

While some of the initial long-course CRT trials utilized bolus 5FU, continuous infusion 5FU is considered a modern standard primarily from clinical improvements seen in the adjuvant setting. ${ }^{19}$ Multiple trials have integrated additional or alternative chemotherapeutics in an effort to improve response rates and clinical outcomes (see Table 3). Capecitabine, an oral 5FU prodrug, has been proposed as an alternative to infusional 5FU, allowing for convenient oral daily dosing. A randomized trial in Germany compared capecitabine to 5FU as the concurrent radiosensitizer and adjuvant chemotherapeutic. A trend for higher PCR and a significant downstaging effect was seen in favor of capecitabine-based CRT. ${ }^{20}$ While LR rates were similar, there were improvements in DM and OS in the capecitabine arm. The results of this trial are somewhat more difficult to interpret, as it included both patients treated with neoadjuvant or postoperative CRT and different adjuvant chemotherapy regimens. Recently reported results from NSABP R-04 suggest similar outcomes with the addition of capecitabine or continuous infusion 5FU to radiation, indicating capecitabine is a standard of care in this setting. ${ }^{21}$ 
The German CAO/ARO/AIO-04 trial sought to integrate oxaliplatin into both the preoperative and adjuvant fluoropyrimidine-based platform. pCR rates were modestly improved with oxaliplatin (17 versus $13 \%$ ), with high rates of compliance and acceptable toxicity. ${ }^{22}$ By contrast, results from the Italian STAR-01 and French ACCORD 12/0405-PRODIGE2 trials adding oxaliplatin to fluoropyrimidine-based CRT regimens did not demonstrate improved $\mathrm{PCR}$ rates, with a significant increase in grade 3/4 toxicity rates seen. ${ }^{23,24}$ The NSABP R-04 Intergroup trial initially sought to test the value of capecitabine compared with continuous infusion 5FU, but was later amended to a 2x2 design to also evaluate oxaliplatin as a component of preoperative CRT. Secondary endpoints of $\mathrm{PCR}$, sphincter-sparing surgery, surgical downstaging, and acute toxicity were recently reported. No statistical differences were seen in surgical outcomes between arms, but the addition of oxaliplatin to either fluoropyrimidine regimen significantly increased toxicity rates. ${ }^{21}$ Whether combination therapy with oxaliplatin will improve longterm clinical outcomes remains unclear. Updated results of ACCORD 12/0405-PRODIGE2 found no improvements in DFS or OS, while CAO/ARO/AIO-04 presented at 2014 ASCO suggested a DFS benefit at 3 years. ${ }^{25,26}$

\section{Randomized Comparisons of Short- and Long-course Radiotherapy}

Two published randomized trials have compared short-course RT and long-course CRT (see Table 4). Polish investigators randomized 312 patients with resectable T3/T4 rectal cancers to 50.4 Gy in 28 fractions with concurrent bolus 5FU and leucovorin followed by TME 4-6 weeks later versus short-course RT (25 Gy/5 fractions) followed by TME in 1 week. Compared with long-course CRT, short-course RT resulted in lower rates of early grade $3 / 4$ toxicities ( 3.2 versus $18.2 \%$; $p<0.001$ ) and improved compliance (97.9 versus 69.2\%). ${ }^{27}$ No significant difference was seen in sphincter preservation (the primary study endpoint), although it was not specified prior to treatment which patients would likely require an APR. Downstaging effects including a higher PCR rate $(0.7$ versus $16.1 \%$ ) and lower rates of positive circumferential margins (12.9 versus $4.4 \% ; p=0.017$ ) in the long-course CRT arm. Adjuvant chemotherapy was left to the discretion of the treating physicians and was more commonly given in the short-course arm (46 versus $30 \%$ ). At a median follow-up of 48 months, there was no significant difference in LR (10.6 versus $15.6 \% ; p=0.21$ ), 4-year DFS (58.4 versus $55.6 \%$ ), 4-year OS (67.2 versus $66.2 \%$ ), or severe late toxicity (10 versus $7 \%$ ) between the short and long courses, respectively.

The second trial was conducted by the Trans-Tasman Radiation Oncology Group (TROG) in which 326 patients with T3NO-2 rectal cancers were randomly assigned to 50.4 Gy in 28 fractions with daily continuous infusional 5FU followed by TME 4-6 weeks later versus short-course RT (25 Gy/5 fractions). Unlike the Polish trial, all patients were staged using modern techniques and adjuvant chemotherapy was administered with short and long courses receiving 6 and 4 cycles, respectively. Pathologic downstaging ( 45 versus $28 \%$; $=0.002$ ) and pCR (15 versus $1 \%$ ) rates were more common in patients assigned to long-course CRT. ${ }^{28}$ No significant difference in APR rates were seen in distal $(<5 \mathrm{~cm})$ tumors (79 versus $77 \%$ ). At a median follow-up time of 6 years, no significant difference was seen in 3-year LR (7.5 versus $5.7 \%$ ), 5-year DM (27 versus $30 \%)$, 5-year OS (74 versus $70 \%$ ), or late toxicity rates when comparing short-course with long course, respectively. German investigators have conducted a third trial of similar design to the TROG study and results are eagerly anticipated.

\section{Future Directions}

A potential limitation of the short-course regimen is limited opportunity for tumor downstaging. By allowing a 4-8 week period following radiotherapy (as in the long-course regimen), improved $\mathrm{PCR}$ and downstaging may be seen with short-course RT. The Stockholm III trial was designed to further study these endpoints. Patients with resectable rectal cancer are randomized to one of three arms: short-course RT followed by surgery in 1 week, the same short-course RT followed by delayed surgery 4-8 weeks following treatment completion, or long-course RT followed by surgery in 4-8 weeks. ${ }^{29}$ Another potential treatment approach using the short-course regimen is the integration of neoadjuvant chemotherapy. The RAPIDO trial, also from Sweden, is randomizing patients with locally advanced rectal cancer to preoperative long-course CRT and selective postoperative adjuvant chemotherapy versus short-course RT followed by 6 cycles of capecitabine and oxaliplatin before surgery. ${ }^{30}$

Not all patients are expected to derive clinically meaningful benefit from neoadjuvant treatment. In the Dutch TME trial, short-course RT in stage I and II patients reduced LR, but also put patients at risk for late toxicities including long-term bowel dysfunction. ${ }^{8,9}$ As a result, neoadjuvant therapy is typically reserved for CT3-4 and/or node positive rectal cancer or select cases of earlier stage distal tumors that may be downstaged to a potential sphincter-sparing surgery candidate. Select PT3NO patients have a low risk of LR and may avoid adjuvant CRT. ${ }^{31}$ Staging studies are sometimes inaccurate resulting in possible overtreatment for patients who may have reasonable outcomes with surgery alone. In the German Rectal trial, $18 \%$ of patients in the postoperative arm were found to have early stage disease despite preoperative imaging classification of CT34 or node positive disease. ${ }^{11}$ By contrast, in a series of CT3NO patients treated with neoadjuvant CRT and resection, $22 \%$ had pathologically positive mesorectal nodes. ${ }^{32}$ This number would have been presumably higher without preoperative treatment. Further improvements in imaging techniques may better select patients for neoadjuvant treatment.

Recently, several alternative treatment paradigms have emerged. In an effort to administer systemic chemotherapeutic doses earlier, investigators at Memorial Sloan Kettering Cancer Center conducted a pilot trial of neoadjuvant infusional fluorouracil, leucovorin, and oxaliplatin (FOLFOX) with bevacizumab for clinical stage II-III rectal cancer patients. Chemotherapy was given for six cycles with selective rather than consistent use of chemoradiotherapy for stable/progressive disease. Out of 32 study participants, two did not complete the prescribed six cycles due to toxicity and were treated with neoadjuvant chemoradiation; all patients had an RO resection with a PCR of $25 \%$. $^{33}$ No local failures have occurred after 4 years of follow-up. Other trials, however, have not demonstrated as promising results with a chemotherapy-alone neoadjuvant approach. ${ }^{34}$ The use of preoperative chemotherapy alone in selected patients is now being evaluated in the Preoperative Radiation or Selective Preoperative Radiation and Evaluation Before Chemotherapy and TME (PROSPECT) trial.

Another approach includes the omission of surgery in clinical complete responders to neoadjuvant CRT. One single institution's experience has been 
reported in patients with CT2-T4 and/or node positive disease who were observed following neoadjuvant CRT if they had no clinical, endoscopic, or radiographic evidence of disease. Approximately half of patients achieved a complete clinical response, but $30 \%$ of these patients later developed a local or pelvic recurrence. ${ }^{35}$ The majority of these recurrences were salvaged with surgery with an overall sphincter preservation rate of $86 \%$. Experience at the Memorial Sloan Kettering Cancer Center has shown similar results in patients having a complete clinical response to neoadjuvant therapy. Out of 32 patients treated with nonoperative intent, six patients developed a local failure. ${ }^{36}$ All local failures were salvaged successfully, avoiding rectal resection in $81 \%$ of these patients. Compared with patients achieving a $\mathrm{PCR}$, DFS, and OS were similar. These retrospective data have been corroborated in a single-institution prospective study from The Netherlands. With an intensive follow-up policy including modern imaging techniques, patients undergoing a 'wait-and-see' policy had less bowel dysfunction and equivalent DFS and OS compared with a control cohort obtaining a PCR after neoadjuvant CRT and TME. ${ }^{37}$

\section{Conclusions}

Two neoadjuvant radiation platforms have been successful in improving outcomes in rectal cancer patients. In northern Europe, the short-course $\mathrm{RT}$ regimen is applied to most patients with rectal cancer, while the longcourse CRT regimen is typically reserved for more advanced cases in the US and other part of Europe. Direct comparisons of the two techniques have not shown statistically significant differences in local control or OS outcomes, although long-term follow-up is needed due to known late toxicity risks associated with the short-course regimen as well as propensity of rectal cancer for late relapse. Ongoing trials should help clarify the optimal timing of surgery and sequencing of chemotherapy and radiation for each approach.
1. Stockholm Rectal Cancer Study Group, Preoperative short-term radiation therapy in operable rectal carcinoma. A prospective randomized trial, Cancer, 1990;66:49-55.

2. Cedermark $B$, Johansson $H$, Rutqvist $L E$, Wilking $N$, The Stockholm I trial of preoperative short term radiotherapy in operable rectal carcinoma A prospective randomized trial. Stockholm colorecta Cancer Study Group, Cancer, 1995:75:2269-75.

3. Stockholm Colorectal Cancer Study Group, Randomized study on preoperative radiotherapy in rectal carcinoma, Ann Surg Oncol, 1996;3:423-30.

4. Folkesson J, Birgisson $H$, Pahlman L, et al., Swedish Rectal Cancer Trial: Iong lasting benefits from radiotherapy on survival and local recurrence rate, I Clin Oncol, 2005;23:5644-50.

5. Birgisson $\mathrm{H}$, Pahlman L, Gunnarsson U, et al., Adverse effects of preoperative radiation therapy for rectal cancer: long-term follow-up of the swedish Rectal Cancer Trial, / Clin Oncol, 2005:23:8697-705.

6. Birgisson H, Pahlman L, Gunnarsson U, Glimelius B, Late gastrointestinal disorders after rectal cancer surgery with and without preoperative radiation therapy, Br I Surg, 2008;95:206-13

7. Carlsen E, Schlichting E, Guldvog I, et al., Effect of the introduction of total mesorectal excision for the treatment of rectal cancer, Br J Surg, 1998:85:526-9.

8. van Gijn W, Marijnen CAM, Nagtegaal ID, et al., Preoperative radiotherapy combined with total mesorectal excision for resectable rectal cancer: 12-year follow-up of the multicentre, randomised controlled TME trial, Lancet Oncol, 2011;12:575-82

9. Peeters KC, van de Velde CJ, Leer JW, et al., Late side effects of short-course preoperative radiotherapy combined with total mesorectal excision for rectal cancer increased bowe dysfunction in irradiated patients-a Dutch colorectal cancer group study, I Clin Oncol, 2005:23:6199-206.

10. Sebag-Montefiore D, Stephens RJ, Steele R, et al., Preoperative radiotherapy versus selective postoperative chemoradiotherapy in patients with rectal cancer (MRC CRO7 and NCIC-CTG C016): a multicentre, randomised trial, Lancet, 2009;373:811-20.

11. Sauer R, Becker $\mathrm{H}$, Hohenberger W, et al., Preoperative versus postoperative chemoradiotherapy for rectal cancer, N Eng/ J Med, 2004:351:1731-1740.

12. Sauer R, Liersch T, Merkel $\mathrm{S}$, et al., Preoperative versus postoperative chemoradiotherapy for locally advanced rectal cancer: results of the German CAO/ARO/AIO-94 randomized phase III trial after a median follow-up of 11 years, I Clin Oncol 2012;30:1926-33.

13. Roh MS, Colangelo LH, O'Connell MJ, et al., Preoperative multimodality therapy improves disease-free survival in patients with carcinoma of the rectum: NSABP R-03, J Clin Oncol, 2009;27:5124-30.
14. Park JH, Yoon SM, Yu CS, et al., Randomized phase 3 trial comparing preoperative and postoperative chemoradiotherapy with capecitabine for locally advanced rectal cancer, Cancer, 2011;117:3703-12.

15. Bosset JF, Calais G, Mineur L, et al., Enhanced tumorocidal effect of chemotherapy with preoperative radiotherapy for rectal cancer: preliminary results-EORTC 22921, J Clin Oncol, 2005:23:5620-7.

16. Bosset JF, Calais G, Mineur L, et al., Fluorouracil-based adjuvant chemotherapy after preoperative chemoradiotherapy in rectal cancer: long-term results of the EORTC 22921 randomised study, Lancet Oncol, 2014;15:184-90.

17. Gerard JP, Conroy T, Bonnetain F, et al., Preoperative radiotherapy with or without concurrent fluorouracil and leucovorin in T3-4 rectal cancers: results of FFCD 9203, J Clin Oncol, 2006:24:4620-5.

18. Braendengen $\mathrm{M}$, Tveit KM, Berglund $\mathrm{A}$, et al., Randomized phase III study comparing preoperative radiotherapy with chemoradiotherapy in nonresectable rectal cancer, J Clin Oncol 2008;26:3687-94

19. O'Connell MJ, Martenson JA, Wieand HS, et al., Improving adjuvant therapy for rectal cancer by combining protractedinfusion fluorouracil with radiation therapy after curative surgery, N Eng/ J Med, 1994;331:502-7.

20. Hofheinz R-D, Wenz F Post S, et al, Chemoradiotherapy with capecitabine versus fluorouracil for locally advanced rectal cancer: a randomised, multicentre, non-inferiority, phase 3 trial, Lancet Oncol, 2012;13:579-88.

21. O'Connell MJ, Colangelo LH, Beart RW, et al., Capecitabine and oxaliplatin in the preoperative multimodality treatment of rectal cancer: surgical end points from National Surgical Adjuvant Breast and Bowel Project Trial R-04, I Clin Oncol, 2014:32:1927-34.

22. Rodel $\mathrm{C}$, Liersch $\mathrm{T}$, Becker $\mathrm{H}$, et al., Preoperative chemoradiotherapy and postoperative chemotherapy with fluorouracil and oxaliplatin versus fluorouracil alone in locally advanced rectal cancer: initial results of the German CAO/ARO/ AlO-04 randomised phase 3 trial, Lancet Oncol, 2012;13:679-87.

23. Aschele C, Cionini L, Lonardi S, et al., Primary tumor response to preoperative chemoradiation with or without oxaliplatin in locally advanced rectal cancer: pathologic results of the STAR01 randomized phase III trial, J Clin Oncol, 2011:29:2773-80.

24. Gerard JP, Azria D, Gourgou-Bourgade S, et al., Comparison of two neoadjuvant chemoradiotherapy regimens for locally advanced rectal cancer: results of the phase III trial ACCORD 12/0405-Prodige 2, J Clin Oncol, 2010;28:1638-44.

25. Gerard JP, Azria D, Gourgou-Bourgade S, et al., Clinical outcome of the ACCORD 12/0405 PRODIGE 2 randomized trial in rectal cancer, I Clin Oncol, 2012;30:4558-65.
26. Rodel C, Liersch T, Fietkau R, et al, Preoperative chemoradiotherapy and postoperative chemotherapy with 5-fluorouracil and oxaliplatin versus 5-fluorouracil alone in locally advanced rectal cancer: Results of the German CAO/ARO/AIO-04 randomized phase III trial, J Clin Oncol, 2014;32:(Suppl. 15); abstr 3500

27. Bujko K, Nowacki MP, Nasierowska-Guttmejer A, et al., Longterm results of a randomized trial comparing preoperative short-course radiotherapy with preoperative conventionally fractionated chemoradiation for rectal cancer, Br J Surg, 2006;93:1215-23

28. Ngan SY, Burmeister B, Fisher RJ, et al., Randomized trial of short-course radiotherapy versus long-course chemoradiation comparing rates of local recurrence in patients with $\mathrm{T} 3$ rectal cancer: Trans-Tasman Radiation Oncology Group trial 01.04, J Clin Oncol, 2012:30:3827-33.

29. Pettersson D, Cedermark $B$, Holm T, et al., Interim analysis of the Stockholm III trial of preoperative radiotherapy regimens for rectal cancer, Br J Surg, 2010;97:580-7.

30. Nilsson PJ, van Etten B, Hospers GA, et al., short-course radiotherapy followed by neo-adjuvant chemotherapy in locally advanced rectal cancer-the RAPIDO trial, BMC Cancer, 2013:13:279

31. Willett $C G$, Badizadegan $K$, Ancukiewicz $M$, Shellito $P C$ Prognostic factors in stage T3NO rectal cancer: do all patients require postoperative pelvic irradiation and chemotherapy?, Dis Colon Rectum, 1999;42:167-73.

32. Guillem JG, Diaz-Gonzalez JA, Minsky BD, et al., CT3NO rectal cancer: potential overtreatment with preoperative chemoradiotherapy is warranted, I Clin Oncol, 2008;26:368-73.

33. Schrag D, Weiser MR, Goodman KA, et al., Neoadjuvant chemotherapy without routine use of radiation therapy for patients with locally advanced rectal cancer: a pilot trial, J Clin Oncol, 2014;32:513-8.

34. Ishii Y, Hasegawa H, Endo T, et al., Medium-term results of neoadjuvant systemic chemotherapy using irinotecan, 5 -fluorouracil, and leucovorin in patients with locally advanced rectal cancer, Eur J Surg Oncol, 2010;36:1061-5.

35. Habr-Gama A, Gama-Rodrigues J, Sao Juliao GP, et al., Local recurrence after complete clinical response and watch and wait in rectal cancer after neoadjuvant chemoradiation: impact of salvage therapy on local disease control, Int I Radiat Oncol Biol Phys, 2014;88:822-8.

36. Smith JD, Ruby JA, Goodman KA, et al., Nonoperative management of rectal cancer with complete clinical response after neoadjuvant therapy, Ann Surg, 2012;256:965-72.

37. Maas M, Beets-Tan RG, Lambregts DM, et al., Wait-and-see policy for clinical complete responders after chemoradiation for rectal cancer, J Clin Oncol, 2011:29:4633-40. 